\title{
Female Genital Tuberculosis
}

\author{
S Ahmed ${ }^{1}$, DR Shaha ${ }^{2}$, P Begum ${ }^{3}$, T Akter $^{4}$
}

\begin{abstract}
:
The incidence of genital tuberculosis varies widely with the social status of the patient and her environment. The incidence is about $1 \%$ amongst gynecological patients attending the outpatient department in the developing country. Genital TB may be asymptomatic and the majority of women are diagnosed during investigations of infertility. Genital system TB represents $15-20 \%$ of extrapulmonary TB and is usually asymptomatic affecting mainly young women in the reproductive age group. Here we presented a case of genital TB which was diagnosed during laparotomy. After confirmation of diagnosis by histopathology, the case was successfully managed by anti-tubercular therapy for 9 months.
\end{abstract}

Key words: Genital Tuberculosis.

\section{Introduction:}

Tuberculosis is a worldwide disease with greater prevalence where the population is concentrated in areas with poor sanitation and unfavorable social and economic condition. Genital TB is one form of extra pulmonary TB and is not uncommon. The incidence is about $1 \%$ among gynecological patients attending the outpatient department in the developing countries ${ }^{1}$. The causative organism is $\mathrm{M}$. tuberculosis human type, in majority.

Genital TB occurs mostly secondary to pulmonary tuberculosis commonly by the haematogenous route in a manner similar to spread to other extra pulmonary sites like urinary tract, bones and joints etc.

The fallopian tubes are affected in almost $100 \%$ of cases followed by the endometrium in $50 \%$, ovaries in $20 \%$, cervix in $5 \%$ and vulva and vagina in $1 \%$ cases.

1. Dr. Shahana Ahmed, FCPS (Obst and Gynae), Assistant Professor, Department of Obstetrics and Gynaecology, Diabetic Association Medical College, Faridpur.

2. Prof. Dr. Dipti Rani Shaha, FCPS (Obst and Gynae), Professor \& Head, Department of Obstetrics and Gynaecology, Diabetic Association Medical College, Faridpur.

3. Dr. Poly Begum, FCPS (Obst and Gynae), Assistant Professor, Department of Obstetrics and Gynaecology, Diabetic Association Medical College, Faridpur.

4. Dr. Tahmina Akter, MD (Paediatrics), Associate Professor, Department of Paediatrics, Diabetic Association Medical College, Faridpur.

Address of correspondence :

Dr. Shahana Ahmed, FCPS (Obst and Gynae), Assistant Professor, Department of Obstetrics and Gynaecology, Diabetic Association

Medical College, Faridpur. Mob:. +88-01714105201,

E-mail: dr.shamolee42rmc@gmail.com
The initial site of infection is in the submucosal layer of the ampullary part of the tube. Direct inoculation of tubercle bacilli can also take place over vulva or vagina during sexual intercourse with a partner suffering from tuberculous lesion of genitalia.

The infection is restricted mostly ( $80 \%)$ to childbearing period (20-40 years). Genital tuberculosis occurs in 10$20 \%$ of patients who have pulmonary tuberculosis in adolescence. The symptoms of genital tuberculosis are infertility, menstrual abnormality, amenorrhoea or oligomenorrhoea, chronic pelvic pain, tubo-ovarian mass, vaginal discharge in the form of post-coital bleeding or blood stained discharge and constitutional symptoms such as loss of weights, malaise, anorexia, pyrexia and anemia etc.

\section{Case report :}

Luchita, 16 years old unmarried young girl presented at outpatient department of Diabetic Association Medical College Hospital with the complaints of abdominal pain, generalized weakness and per vaginal whitish discharge. Her menstrual cycle was regular with average flow and duration. Her age of menarche was at 13 years. Her grandfather suffered from TB 2 years back. On examination her weight was $47 \mathrm{~kg}$, BP$100 / 70 \mathrm{mmHg}$. On per abdominal examination there was a lump $8 \times 10 \mathrm{~cm}$ in diameter, occupying partly left iliac, left lumber, umbilical and hypogastrium region. Margin was irregular, smooth surface, firm in consistency and mobility was restricted. She was admitted for further evaluation with a plan of 
laparotomy. Her USG report revealed thick wall septed cyst with floating low level internal echoes $(9.9 \mathrm{~cm} \times 5.5 \mathrm{~cm}$; approx. vol. $132 \mathrm{ml})$ noted in pelvic region. Her CA-125 was $98.5 \mu / \mathrm{ml}$, chest $\mathrm{x}$-ray was normal. Other investigations show no abnormality.

Her laparotomy was done. After opening of abdomen there was dense adhesion of left adnexa with surrounding structures (intestine and omentum). Adhesiolysis was done as per as possible. After taking biopsy and peritoneal toileting abdomen was closed in layers.

Histopathology report showed granulomatous inflammation of ovarian tissue. The granuloma was composed of epitheloid cells containing Langerhan's type of giant cell.

The treatment consists of Isoniazid plus Rifampicin plus Ethambutol plus Pyrazinamide orally. In addition pyridoxin $300 \mathrm{mg}$ a day was also administered to prevent neuropathy. Antitubercular therapy was continued for 9 months and hepatic and renal function tests and periodical eye fundus, visual acuity and visual fields examinations were normal during follow up.

\section{Discussion :}

Although genital TB can occur in any age group, the majority of patients are in the reproductive age group ${ }^{1}$. Seventy five percent of them being in the 20-45 years $\mathrm{age}^{2}$. Postmenopausal women accounts for $7-11 \%$ of cases of genital TB.

Genital TB may be asymptomatic and the majority of women are diagnosed during investigations of infertility ${ }^{2}$. Constitutional symptoms of weight loss, feeling unwell and night sweats may resemble classical acute pelvic inflammatory disease (PID) ${ }^{3} \mathrm{M}$. tuberculosis is the causative organism in $90-95 \%$ of cases whereas Mycobacterium bovis can also cause the disease $^{4}$.

Infection of the female genital tract with $\mathrm{TB}$ is relatively rare entity. There are two main routes for the infection, the first is blood borne from a primary site such as the lungs, or kidneys. The other route is through sexual intercourse where the infection can manifest itself as local lesions. Infection of the vulva, vagina and cervix can be caused by direct inoculation in the lower female reproductive tract if the sexual partner has genital and/or urinary TB infection. In that respect genital TB can be considered as sexually transmitted disease ${ }^{5}$.

Endometrial TB and involvement of the fallopian tubes are obvious explanations in relation to infertility as these conditions can interfere with implantation and sperm/zygote transport. In a study concerning the effect of genital TB on fertility hysterosalpingogram (HSG) revealed tubal abnormalities in $17(95.2 \%)$ out of 21 patients. The most common abnormality was a bilateral tubal blockage and deformity of the uterine cavity was found in $54.4 \%$ of cases $^{6}$. TB can also affect the ovaries and cervix and this may have an impact on fertility as well. Another important manifestation of genital TB is menstrual irregularities. Irregularities may include oligomenorrhoea or amenorrhoea. An important pathological finding in women with genital TB in Asherman's syndrome that explains the menstrual irregularities and infertility as well. Intra and postoperative complications are increased after surgery in women with genital $\mathrm{TB}^{7}$.

General, abdominal and pelvic examination findings are normal in majority of genital tuberculosis patients ${ }^{8,9}$. A pelvic mass may be identified and adnexal tenderness may be elicited. Diagnosis is achieved most effectively through a combination of a high index of suspicion especially in areas of low prevalence through initial clinical assessment and appropriate investigations. High risk factors include a history of previous pulmonary TB infection, contact with a pulmonary TB sufferer, recent travel to or migration from high prevalence countries ${ }^{10}$. Chest X-ray is aimed at demonstrating current or past TB lesions in the lungs, which are a common site for primary infection. However, most chest X-ray are normal ${ }^{11}$.

Whenever feasible, every effort should be made to send specimens and tissue for culture, in order to confirm diagnosis and establish drug sensitivities. Suspected tuberculous lesions in accessible sites such as the vagina, cervix and the vulva may be biopsied directly. Endometrial tissue may be obtained by aspiration biopsy or dilatation and curettage or directly at hysteroscopy. Endometrial biopsy is best performed in the premenstrual period. Menstrual fluid can be obtained from the vagina during the first day of menstruation for culture and microscopy ${ }^{12}$.

Histology demonstrates the typical caseous granulomatous lesion with giant epitheloid cells. Rapid nucleic acid amplification techniques such as polymerase chain reaction (PCR) allow direct identification of $\mathrm{M}$. tuberculosis in clinical specimens. Such methods can detect fewer than ten organisms in clinical specimens compared with 10000 necessary for smear positivity. Although PCR is more sensitive (8595\%) than microscopy and bacteriological culture on pulmonary specimens. It does not distinguish live from killed bacilli ${ }^{11,12}$. Abdominal and pelvic ultrasound, computed tomography (CT) and magnetic resonance imaging (MRI) are employed in circumstance where an abdominal or pelvic mass is present ${ }^{13}$. 


\section{Conclusion :}

The incidence of female genital TB is increasing. So gynecologists will be increasingly faced with cases of TB and its consequences. Genital TB may be asymptomatic or may present with atypical symptoms or mimic other conditions. It is imperative to consider the possibility of TB in women in the reproductive age group, who present with the symptoms of infertility, chronic pelvic pain and menstrual dysfunction, where other causes have been excluded. This is particularly important when these women fall into the high risk category for TB infection.

\section{References :}

1. Has DW. Mycobacterial diseases. In: Mandell GL, Bennet JE, Dolin R, editors. Principles of Practice of Infectious Diseases. Philadelphia, PA: Churchill Livingstone 2000; p. 2576-607.

2. Aka N,Vural TZE. Evaluation of patients with active pulmonary tuberculosis for genital involvement. J Obsetet Gynaecol Res. 1997; 23:337-40.

3. Rajamaheswari N. Pelvic tuberculosis. Int J Microbiol.2008; 27:361-3.

4. Cow TWP, Lim KB, Valliparim S. The masquerades of female pelvic tuberculosis: case reports and review of literature on clinical presentation and diagnosis. J Obstet Gynaecol Res. 2002; 28:203-10.

5. Chowdhury NN. Overview of tuberculosis of the female genital tract. J Indian Med Assoc. 1996; 94:345-6.

6. Lamba H, Byrne M, Goldin R, Jenkins C. Tuberculosis of the cervix: case presentation and a review of the literature. Sex Transm Inf. 2002; 78:62-3.

7. Qureshi RN, Samad S, Hamid R, Lakha SF. Female genital tuberculosis revisted. J Pak Med Assoc. 2001; 51:16-18.

8. Gaur BM, Meheshwari B, Lal N. Tuberculosis endometritis: a clinico-pathological study of 1000 cases. Br J Obstet Gynaecol. 1983; 90:84-6.

9. Samal S, Gupta U, Agarwal P. Menstrual disorders in genital tuberculosis. J Indian Med assoc. 2000; 98:126-7.

10. Muechler E, Minkowitz S. Postmenopausal endometrial tuberculosis. Obsetet Gynaecol. 1971; 38:768-70.

11. Health protection agency. Preliminary Annual Report on Tuberculosis Reported in 2002 England, Wales and Northern Ireland. London: HPA; 2004 [www.hpa.org.uk/infections].

12. Rose AMC, Sinka K, Watson JM, Mortimer JY, Charlett A. An estimate of the contribution of HIV infection to the recent rise in tuberculosis in England and Wales. Thorax 2002; 57:442-5.

13. Raut VS, Mahashur AA, Sheth SS. The Mantoux test in the diagnosis of genital tuberculosis in women. Int J Gynaecol Obstet. 2001; 72:165-9. 